\title{
RECENT RESTORATION OF SKIP-STOP OPERATION IN THE KOREAN URBAN RAILWAY
}

\author{
SUK-MUN OH, KYUNG-MIN KIM, HAG-LAE RHO \& KEUN-YUL YANG \\ Korea Railroad Research Institute, Korea
}

\begin{abstract}
This paper addresses recent restoration of skip-stop operation in the Korean urban railway. Most urban railways in Korea were constructed without consideration of skip-stop operation as many other foreign urban railways. The Korean government had been requested to improve service quality, especially in operational speed, and it announced a policy to restore skip-stop operation for the urban railway lines. This paper presents roles of a central government for restoration of skip-stop operation. Besides leading by research and development side in technical solutions, the roles of the government are very important to implement the restoration. As examples of government's roles, this paper addresses Korean government's recent policy to restore the skip-stop operations.
\end{abstract}

Keywords: restoration, skip-stop, express train, urban railway.

\section{INTRODUCTION}

Many researchers have published their works on the skip-stop operation in the urban railway. Suh et al. [1] focuses on A/B type skip-stop operation problem. It presents a simulation method to deal with the problem for an instance of Line 5 in Seoul Korea.

Freyss et al. [2] focuses on A/B type skip-stop optimization problem similarly to Suh et al [1]. It tackles the problem with a continuous approximation approach. The problem is described with a set of geographically dependent continuous parameters like the density of stations for a given line.

Niu [3] focuses on how to determine the skip-stop scheduling for a congested urban transit line during the morning rush hours. It presents a nonlinear programming model and a bilevel genetic algorithm to solve the model. The algorithm searches possible departure times of vehicles at the terminal by the outer and the skip-stop operations by the inner genetic algorithms.

Cao et al. [4] present a comprehensive estimation model for the skip-stop operation strategy. It established a 0-1 integer programming formulation and a tabu search algorithm to deal with formulation. It presents results of an example of a Chinese urban rail transit network.

Tan et al. [5] deal with the urban rail train stop schedule problem in order to better serve the transport demand. It presents a bilevel modelling similarly to Niu [3] but mathematic programming model with a game theory relation between the two levels differently. It presents an example of ChongQing urban rail line 2 in Chinese urban railway by using the solution approach.

However, all research introduced above, except Suh et al. [1], deal with the optimization problem of skip-stop operation for a newly construction. This paper deals with restoration of the skip-stop operation for an existing infrastructure and E \& $\mathrm{M}$ systems. Moreover, the previous research handles the problem within academic point of view, but this paper addresses the problem with a decision-making process in government point of view.

The first modernized Korean urban railway (Line \#1) started the operation in the capital area 1974. After that, seven lines (Lines \#2-8) were opened by 1999 in the city. All the eight 
lines were constructed without consideration of skip-stop operation as many other foreign urban railways.

The first skip-stop operation was implemented in Line \#9 which was constructed in 2009, almost 10 years later than the Line \#8. Line \#9 was welcomed by citizens who could use the first skip-stop service and interested by researchers who could observe behaviour of the operation. Most passengers in Line \#9 prefer express train than normal train. The reason of the preference is simple that the express train provides faster service than the normal train.

Fig. 1 shows congestion rates of four stations in Line \#9. Red and blue bars represent congestion rates of express and normal trains, respectively. Express trains place in parts of higher congestion rate than normal trains in the four stations. The higher congestion rate is originated from the higher number of passengers chosen the express train in spite of its inconvenience. The passengers are willing to tolerate the inconvenience for arriving their offices in time. This means citizens much prefer the express trains to the normal trains, particularly in morning peak.

Beside the comparisons of congestion rates, the social policy division in OECD published "Average time spent travelling to and from work between 1999 to 2014" in 2016 as shown in Fig. 2. The Korean time spent is fifty-eight min. which is almost twice the OECD average of twenty-eight min. We believe long time spent for work makes quality of life worse.

Under consideration of the preference to express trains as well as long time spent for work, the Korean government set a policy to restore skip-stop operation for the urban railway lines to cut down the long time spent for work.

This paper introduces the policy for restoration of skip-stop operation and addresses technical points should be tackled during implementation of restoration. Moreover, it comments some missing points that were not covered by the policy.

Later of this paper comprised; section two addresses a policy for restoration of skip-stop operation in Korea. Last, section three is for summary.
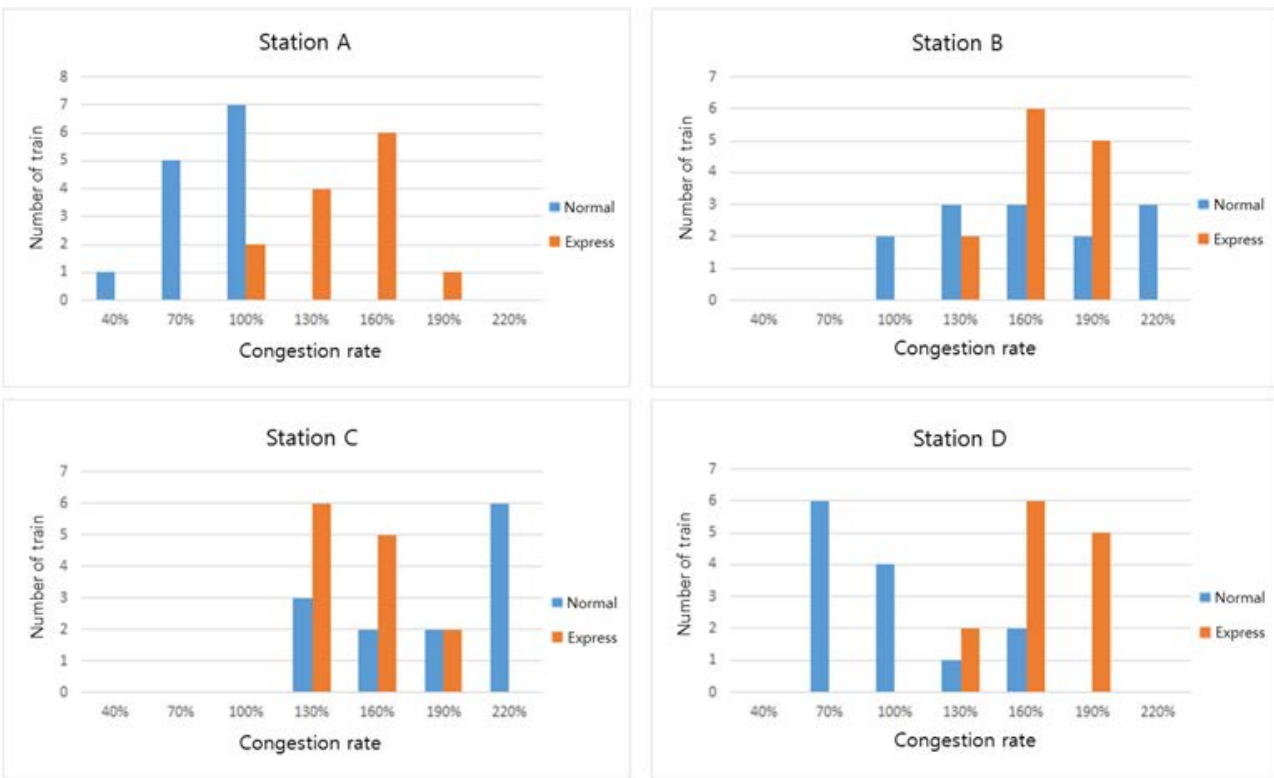

Figure 1: Comparisons of congestion rate in Seoul Metro Line \#9. 
Chart LMF2.6.A: Average time spent travelling to and from work, 1999-2014 Time spent travelling to and from work or study, in minutes per day, all 15-to-64-year-olds ${ }^{\mathrm{b}}$

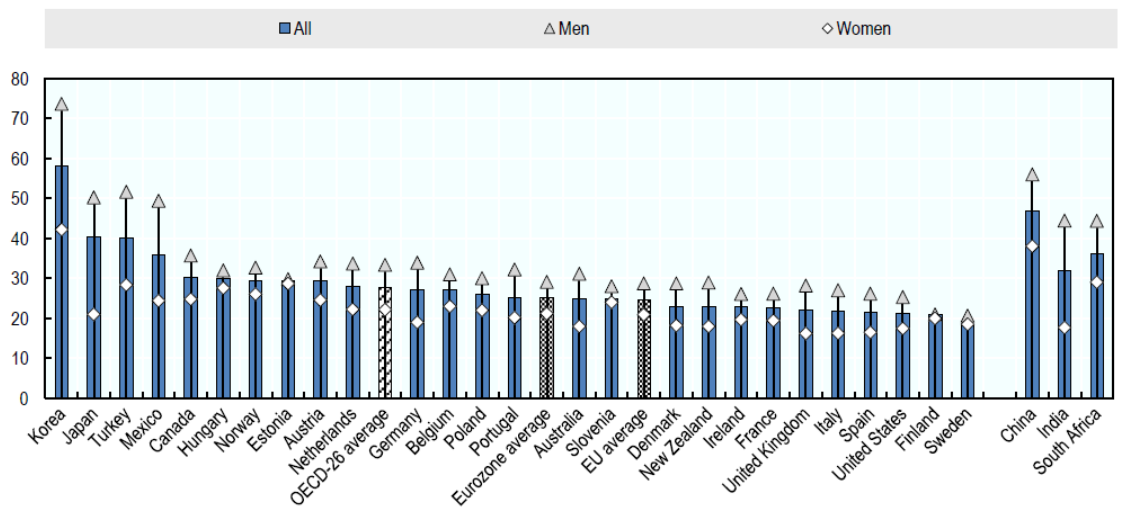

Countries are ranked by time spent to and from work or study for all men and women

a) Australia: 2006; Austria: 2008-09; Belgium: 2005; Canada: 2010; China: 2008; Denmark: 2001; Estonia: 2009-10; Finland: 2009-10; France: 2009; Germany: 2001-02; Hungary: 1999-2000; India: 1999; Italy: 2008-09; Ireland: 2005; Japan: 2011; Korea: 2009; Mexico: 2009; the Netherlands: 2005-06; New Zealand: 2009-10; Norway: 2010; Poland: 2003-04; Portugal: 1999; Slovenia: 2000-01; South Africa: 2010; Spain: 2009-10; Sweden: 2010; Turkey: 2006; the United Kingdom: 2005; and the United States: 2014.

b) Except for Australia (15+ year olds), China and Hungary (15-74 year olds) and Sweden (25-64 year olds).

Source: OECD gender data portal (http://www.oecd.org/gender/) and OECD secretariat estimates based on national time-use surveys.

Figure 2: OECD data of average travelling time for working. (Source: OECD [6].)

\section{REQUIRED ROLES AND IMPLEMENTATION POLCY}

This section presents the required roles for a central government to restore the skip-stop operation. Moreover, it presents some examples of implementation policy made by Korean government for the restoration recently. For successful restoration of the skip-stop operation, a central government has to take charge of roles in three different issues at least.

\subsection{Required roles of a central government}

First, the central government has to revise and improve the existing guideline of feasibility study for construction of a new railroad line or reconstruction of an existing railroad line. When express and normal trains operate in a same line, the existing benefit estimation method cannot assess the number of express passengers differentiated from the normal passengers. In addition, the existing method accounts the waiting time to half of the average headway. However, an operation ratio of express vs. normal is set to $1: 2$ or 1:3 frequently. These relatively lower frequency of the express train make the express passengers behave under consideration of the schedule time more than the normal passengers who have relatively higher frequency of service. Therefore, the express passengers' waiting time should be assessed less than the half of the average headway differently from the existing method. In order to estimate the benefit from the skip-stop operation correctly, the central government is recommended to revise and improve their guideline.

Second, the central government has to check the technical feasibility to restore the skipstop operation. If some technical problems have not solved yet, it has to motivate its research institutes to develop the technologies to solve the problems. For example, a railway line, constructed under the surface in depth through a tunnel (not an open-cut box), is fairly difficulty to construct additional side-track for meet/overtake between express and normal trains without interruption of the existing train operation. This technical problem should be 
solved by development of new and safe technologies. The motivation to develop the technologies is a role of the central government.

Third, the central government has to prepare a master plan to restoration of the skip-stop operation. Many mega-cities, including Seoul in Korea, operate the urban railways in network-wide. Thus, a policy of the restoration should be a form of master plan, not be a form of local oriented one. The policy and master plan are recommended to prepare in cooperation with local government as far as possible. The policy and master plan are further recommended to the official long-term plan the urban railway.

The Korean government set a plan for restoration of skip-stop operation in 2017, despite difficult problems in retrofitting infrastructure [7], [8]. This section addresses the plan in three different phases.

\subsection{Extending the existing skip-stop operation}

The first phase is to extend the existing skip-stop operation without considerable construction works. Four lines are categorized to the first phase; Gyeongin, Gyeongui, Suin and Ansan Lines. The Gyeongin line has operated express trains with exclusive express line so far. The government made a plan to launch 18 super-express trains additionally. The super-express trains overtake the existing express trains. The super-express trains reduce 20 min. compare to the normal trains.

The line section where the express trains operate was extended and 10 express trains were increased additionally in the Gyeongui line. The extension of the express line section reduces six min. compare to the existing express trains.

The Suin line got $133 \%$ increase of passenger transportation in 2017 compare to the last year. The line newly launches eight express trains daily. The new express trains may cut down travel time by seven min.

The Ansan line took similar strategy with the Gyeongui line. It extended the express line section and increased eight express trains daily. The improved skip-stop operation scheme makes travel time shorten by $5.5 \mathrm{~min}$.

The first phase of extension of skip-stop operation was already implemented in 2017. It is reported that outcome of the first phase is quite successful. Fig. 3 shows extended line section where the express trains operate and their stops in the section.

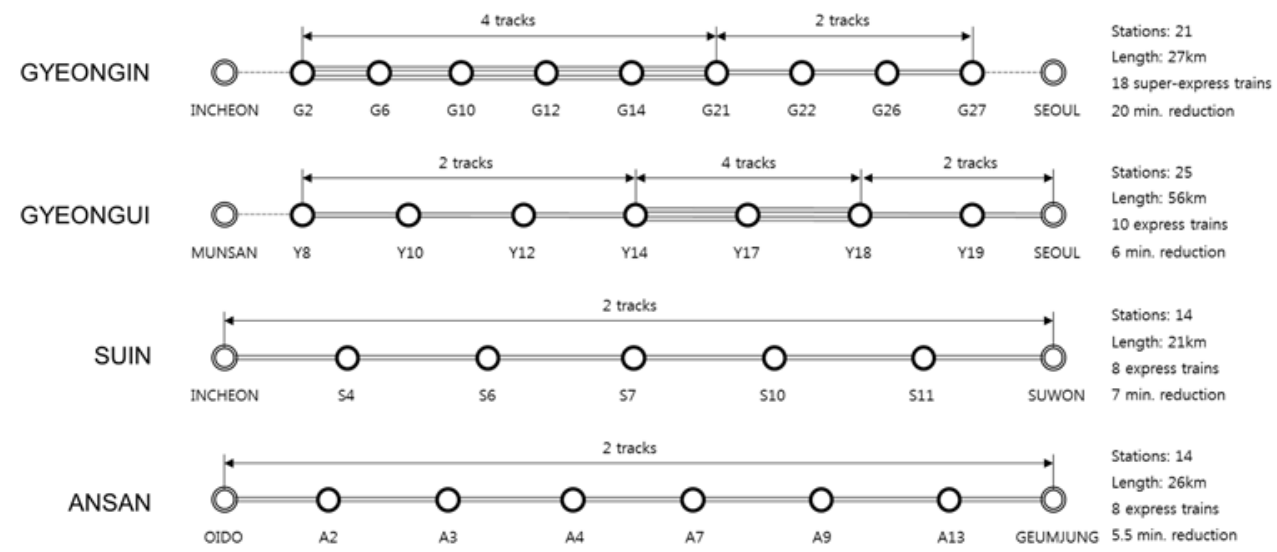

Figure 3: Extending express sections and stops. 


\subsection{Construction of additional side-track}

The second phase is to extend the existing skip-stop operation with construction of additional side-track. Four lines are categorized to the second phase; Gyeongbu, Bundang, Kwachon. Ilsan lines. The Gyeongbu line $(96 \mathrm{~km})$ has a plan to extend existing skip-stop operation scheme by construction of six side-track additionally. Fortunately, all the six side-track will be constructed on the ground. This makes the construction cost and period saved. The project for the Gyeongbu line will begin in this (2018) year and finish by 2020 .

The Bundang line $(53 \mathrm{~km})$ has operated 12 express trains daily for a one-third portion of the total length. The plan for this line is to increase the number of express trains operated daily and extend the express line section to two-thirds by construction of two side-track additionally. The express trains will reduce the travel time by $21 \mathrm{~min}$. compared to the normal trains.

The Kwachon line $(14.4 \mathrm{~km})$ is directly connected to Ansan line which addressed in the first phase. So that express trains operate from Ansan to Kwachon lines directly. In order to implement the direct operation scheme, two side-track will be constructed additionally in the Kwachon line. The travel time will be shortened seven min. by this project.

The Ilsan line $(19.2 \mathrm{~km})$ has a plan to cut down seven min. in travel time by construction of three side-track. The later three lines are under feasibility study project and the new express trains will start operation not later than 2022. Fig. 3 shows the four lines in the plan of the phase two. The block filled circles represent stations where additional side-track to be constructed.

\subsection{Construction of additional side-track}

The third phase is a long-term plan relative to the previous two phases. The third phase is socalled GTX (Great Train eXpress) project. Actually, the GTX project was planned in previous to the skip-stop restoration plan. The GTX project consists of three lines; A - line $(73.7 \mathrm{~km}), \mathrm{B}-$ line $(48.7 \mathrm{~km})$ and C - line $(45.8 \mathrm{~km})$. The GTX trains will operate in $180 \mathrm{~km} / \mathrm{h}$ maximum speed under surface of 40m. Fig. 5 shows the GTX network of the third phase. The GTX trains are quite similar to RER trains of Paris.

Table 1 shows benefits estimation of GTX network. Quite large benefit is estimated in several terms. However, $\mathrm{B} / \mathrm{C}$ ratio of Line $\mathrm{B}$ and $\mathrm{C}$ is lower than one. This is because the construction cost is so huge. Recently, various cost saving methods are under research.

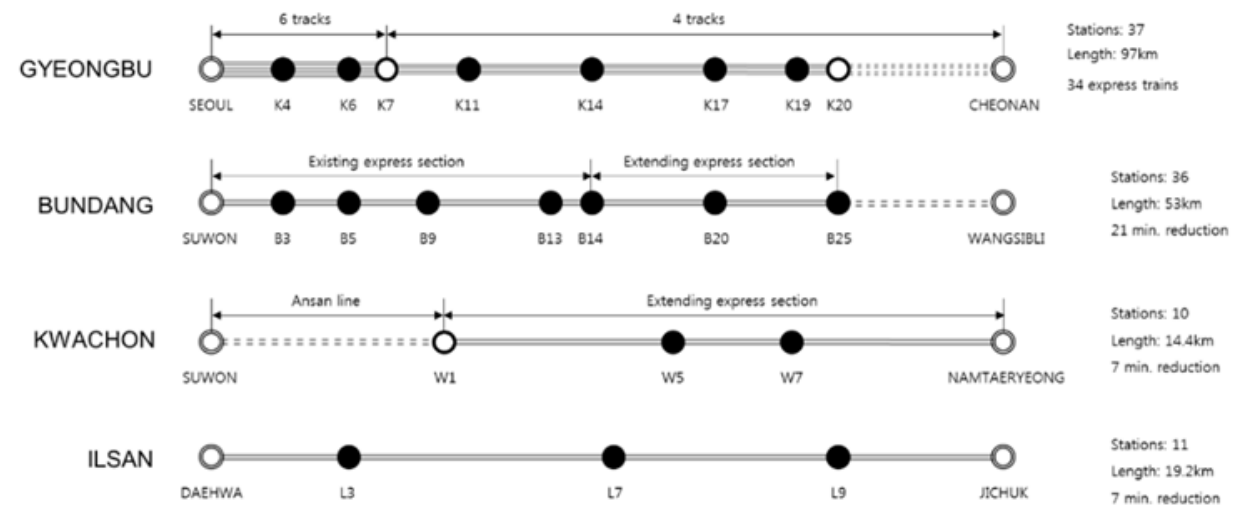

Figure 4: Construction of additional side-track. 


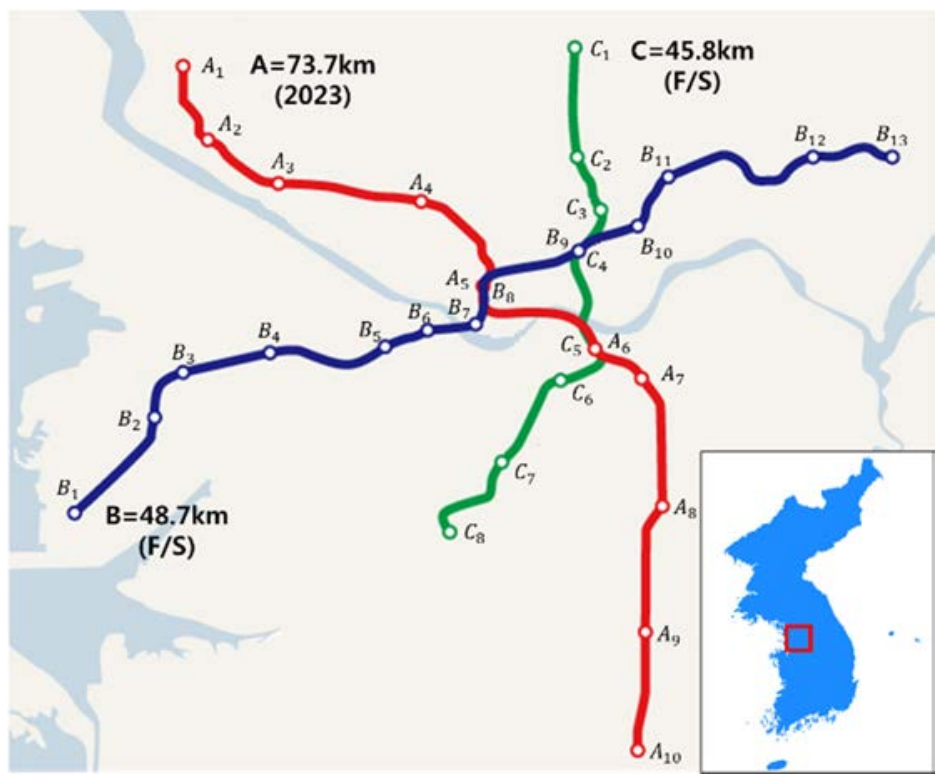

Figure 5: Plan of GTX network.

Table 1: Benefit estimation of GTX network.

\begin{tabular}{|l|l|l|}
\hline Terms & Units $\left(\times 10^{6}\right)$ & Reductions \\
\hline Operational costs & USD/yr & 1,090 \\
\hline Production inducement & USD & 22,727 \\
\hline Job creation & Employees & 0.17 \\
\hline Vehicles inflowing city centre & Veh./yr & 50 \\
\hline $\mathrm{CO}^{2}$ emission & Tons/yr & 0.36 \\
\hline Costs for treatment $\mathrm{CO}^{2}$ & USD/yr & $15 \sim 50$ \\
\hline Energy consumption & Liters/yr & 180 \\
\hline
\end{tabular}

\section{SUMMARY AND CONCLUSIONS}

This paper addresses recent restoration of skip-stop operation in the Korean urban railway together with required roles of central government for successful implementation of the restoration. Particularly, the paper addresses the Korean government's plan to restore the skip-stop operation consists of three phases.

The first phase is to extend the existing skip-stop operation without considerable construction works. Four lines are categorized to the first phase; Gyeongin, Gyeongui, Suin and Ansan Lines. In order to extend the existing skip-stop operation, efficient train scheduling tool is indispensable. This issue has been introduced in Oh and Min [8].

The second phase is to extend the existing skip-stop operation with construction of additional side-track. Four lines are categorized to the second phase; Gyeongbu, Bundang, Kwachon. Ilsan lines. Both the first and second phases are aimed to reduce the travel time 
10-20\% approximately. The construction work for the additional side-track is required to be carried out without halting existing train service. Therefore, the construction work requires safety guaranteed method. This issue has been introduced in Oh et. al. [7].

The third phase, so-called GTX (Great Train eXpress) project, was planned in previous to the skip-stop restoration plan. The GTX project consists of three lines; A - line $(73.7 \mathrm{~km}), \mathrm{B}$ - line $(48.7 \mathrm{~km})$ and C - line $(45.8 \mathrm{~km})$. The GTX trains will operate in $180 \mathrm{~km} / \mathrm{h}$ approximately in the capital area.

\section{REFERENCES}

[1] Suh, W.H., Chon, K.S. \& Rhee, S.M., Effect of skip-stop policy on a Korean subway system. Transportation Research Record, 1793(02-3434), pp. 33-39, 2002.

[2] Freyss, M., Giesen, R. \& Munoz, J.C., Continuous approximation for skip-stop operation in rail transit. Porcedia - Social and Behavioural Science, 80, pp. 186-210, 2013.

[3] Niu, H., Determination of the skip-stop scheduling for a congested transit line by bilevel genetic algorithm. International Journal of Computational Intelligence Systems, 4(6), pp. 1158-1167, 2011.

[4] Cao, Z.C., Yuan, Z.Z. \& Li, D.W., Estimation method for a skip-stop operation strategy for urban rail transit in China. Journal of Modern Transportation, 22(3), pp. 174-182, 2014.

[5] Tan, Z.M., Cheng, J., Peng, Q.Y. \& Gan, M., Hybrid stop schedule of urban rail train. Journal of Industrial Engineering and Management, 8(1), pp. 267-279, 2015.

[6] OECD Family Database, LMF2.6: Time spent travelling to and from work. www.oecd.org/els/family/database.htm. Accessed on: 10 Feb. 2018.

[7] Oh, S.M., Kim, K.M., Rho, H.L. \& Yang, K.Y., Retrofitting infrastructure for skip-stop operation urban railways. COMPRAIL, pp. 67-77, 2016.

[8] Oh, S.M. \& Min, J.H., Dr. METRO: a demand-responsive metro-train operation planning program. COMPRAIL, pp. 595-601, 2014. 